\title{
Convolutional Sparse Coding-based Image Decomposition
}

He Zhang

he.zhang92@rutgers.edu

Vishal M.Patel

http://www.rci.rutgers.edu/ vmp93
Electrical and Computer Engineering

Rutgers, the state University of New Jersey NJ, USA

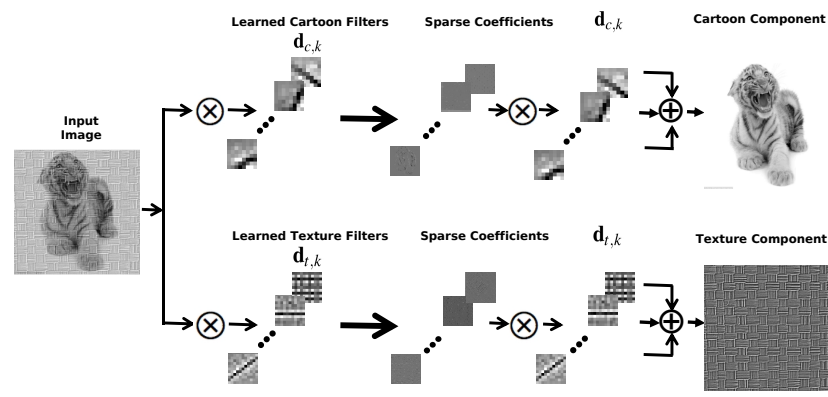

Figure 1: An overview of the proposed CSCD method for image decomposition.

Contribution: Even though dictionary-based image decomposition methods have been proposed in the literature for the purpose of decomposing an image into cartoon and texture parts, most of the existing approaches are patch-based and features learned with these methods often contain shifted versions of the same features [1]. To overcome this issue, we propose a novel sparsity-based method for cartoon and texture decomposition based on Convolutional Sparse Coding (CSC). Our method first learns a set of generic filters that can sparsely represent cartoon and texture type images. Then using these learned filters, we propose a sparsity-based optimization framework to decompose a given image into cartoon and texture components. Figure 1 gives an overview of the proposed images separation method. By working directly on the whole image, the proposed image decomposition algorithm does not need to divide the image into overlapping patches for leaning local dictionaries. Extensive experiments indicate the proposed method performs favorably compared to state-ofthe-art image decomposition methods.

Proposed Method: Following the mixture model

$$
\mathbf{y}=\mathbf{y}_{c}+\mathbf{y}_{t},
$$

given an input image $\mathbf{y}$, our goal is to separate it into a cartoon part $\mathbf{y}_{c}$ and a texture part $\mathbf{y}_{t}$. Assume that we have already learned the convolutional filters corresponding to $\mathbf{y}_{c}$ and $\mathbf{y}_{t}$ by solving the CSC problem for the cartoon and the texture components separately. That is, we have learned $\left\{\mathbf{d}_{c, k}\right\}_{k=1}^{K_{c}}$ and $\left\{\mathbf{d}_{t, k}\right\}_{k=1}^{K_{t}}$ such that $\mathbf{y}_{c}=\sum_{k=1}^{K_{c}} \mathbf{d}_{c, k} * \mathbf{x}_{c, k}$ and $\mathbf{y}_{t}=\sum_{k=1}^{K_{t}} \mathbf{d}_{t, k} * \mathbf{x}_{t, k}$, where $\mathbf{x}_{c, k}$ and $\mathbf{x}_{t, k}$ are the sparse coefficients that approximate $\mathbf{y}_{c}$ and $\mathbf{y}_{t}$ when convolved with the filters $\mathbf{d}_{c, k}$ and $\mathbf{d}_{t, k}$, respectively. We propose to estimate $\mathbf{y}_{c}$ and $\mathbf{y}_{t}$ via $\mathbf{x}_{c, k}$ and $\mathbf{x}_{t, k}$ by solving the following CSC-based optimization problem

$$
\begin{aligned}
\hat{\mathbf{x}}_{c, k}, \hat{\mathbf{x}}_{t, k} & =\arg \min _{\mathbf{x}_{c, k}, \mathbf{x}_{t, k}} \frac{1}{2}\left\|\mathbf{y}-\sum_{k=1}^{K_{c}} \mathbf{d}_{c, k} * \mathbf{x}_{c, k}-\sum_{k=1}^{K_{t}} \mathbf{d}_{t, k} * \mathbf{x}_{t, k}\right\|_{2}^{2} \\
& +\lambda_{c} \sum_{k=1}^{K_{c}}\left\|\mathbf{x}_{c, k}\right\|_{1}+\lambda_{t} \sum_{k=1}^{K_{t}}\left\|\mathbf{x}_{t, k}\right\|_{1}+\beta T V\left(\sum_{k=1}^{K_{c}} \mathbf{d}_{c, k} * \mathbf{x}_{c, k}\right) .
\end{aligned}
$$

Once, $\mathbf{x}_{c, k}$ and $\mathbf{x}_{t, k}$ are estimated, the two components can be obtained by $\hat{\mathbf{y}}_{c}=\sum_{k=1}^{K_{c}} \mathbf{d}_{c, k} * \hat{\mathbf{x}}_{c, k}$ and $\hat{\mathbf{y}}_{t}=\sum_{k=1}^{K_{t}} \mathbf{d}_{t, k} * \hat{\mathbf{x}}_{t, k}$. We propose an iterative method for solving the above optimization problem. The overall CSCD algorithm is summarized in the Algorithm 1 , where $\lambda_{c}$ and $\lambda_{t}$ are the changing parameters corresponding to the two parts, $L$ is the total iteration number.

Experiments: We present the results of our proposed CSCD algorithm for image decomposition and compare them with the sparsity-based MCA method [4], adaptive dictionary learning-based MCA (A-MCA) method [3], and a recent stat-of-the-art Block Nuclear Norm (BNN) based image decomposition method [2]. In these experiments, we use the Peak Signal
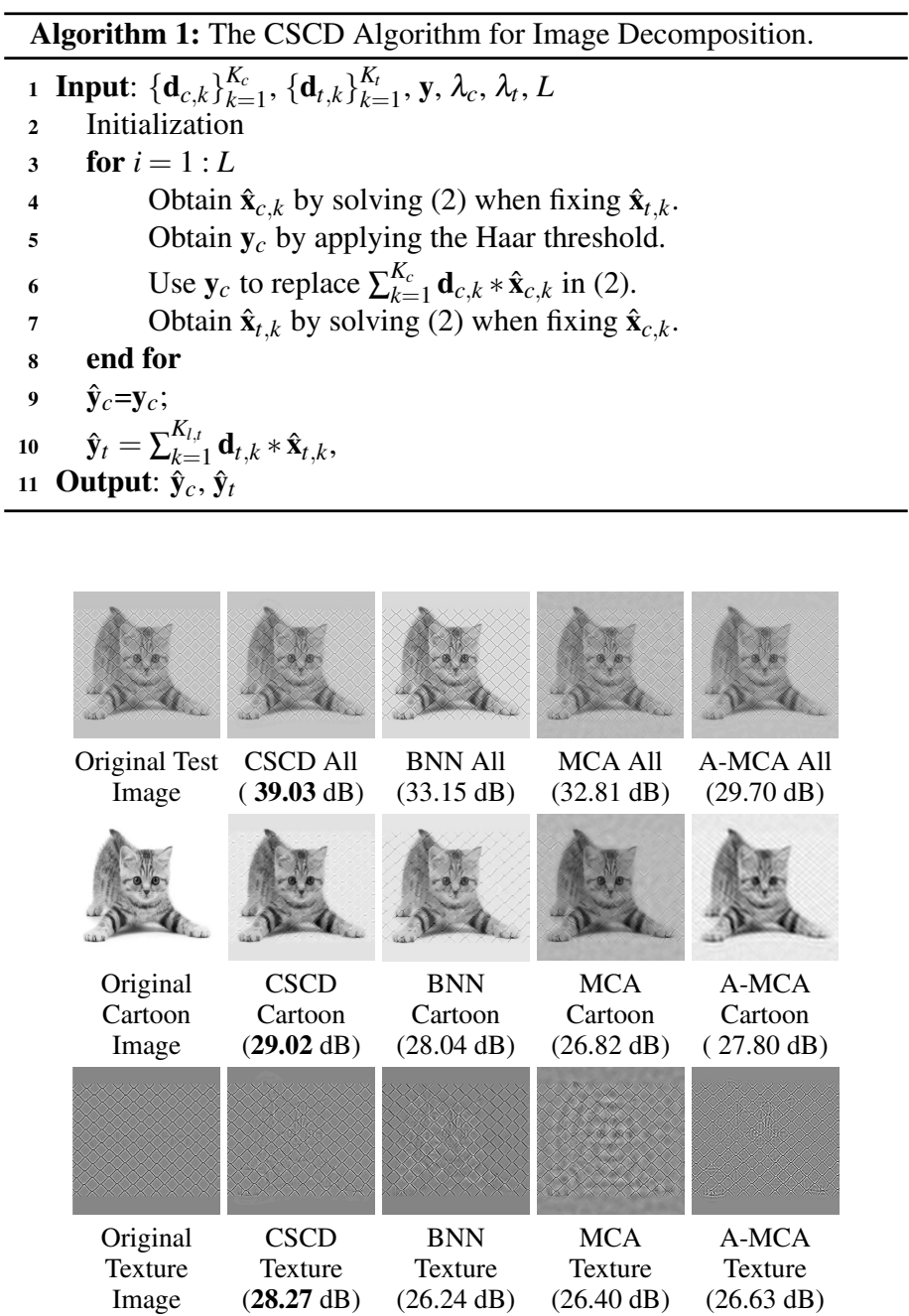

Figure 2: Image decomposition results on the Cat+Cage image. We compare the performance of our method with that of BNN, MCA and A-MCA.

to Noise Ratio (PSNR) to measure the performance of the routines tested (See Figure 2). Various experiments show the significance of our CSCbased image separation method over the other methods.

Aknowledgement: This work was supported by an ARO grant W911NF16-1-0126.

[1] Michael Elad. Sparse and Redundant Representations: From Theory to Applications in Signal and Image Processing. Springer, 1st edition, 2010. ISBN 144197010X, 9781441970107.

[2] Shintaro Ono, Takamichi Miyata, and Isao Yamada. Cartoon-texture image decomposition using blockwise low-rank texture characterization. Image Processing, IEEE Transactions on, 23(3):1128-1142, 2014.

[3] Gabriel Peyré, Jalal Fadili, and Jean-Luc Starck. Learning the morphological diversity. SIAM Journal on Imaging Sciences, 3(3):646$669,2010$.

[4] Jean-Luc Starck, Michael Elad, and David L Donoho. Image decomposition via the combination of sparse representations and a variational approach. Image Processing, IEEE Transactions on, 14(10): 1570-1582, 2005. 